Estudios sobre armas antiguas, arte militar $\mathrm{y}$ vida cultural en oriente y occidente

XXXVI (2016), pp. 161-179

ISSN: 0436-029X

doi: 10.3989/gladius.2016.0008

\title{
“CRUZANDO ACEROS”. EL COMERCIO DE ESPADAS ENTRE ESPAÑA E ITALIA EN LOS SIGLOS XVI Y XVII ${ }^{1}$
}

\author{
“CROSSING STEELS". SWORD TRADE NETWORKS BETWEEN SPAIN AND \\ ITALY IN SIXTEENTH AND SEVENTEENTH CENTURIES
}

POR

\author{
Rafael M. Girón Pascual ${ }^{*}$
}

\section{RESUMEN - ABSTRACT}

Este artículo analiza el comercio de espadas entre España e Italia en los siglos XVI y XVII, por medio del estudio de dos de las rutas comerciales utilizadas por las redes mercantiles europeas, a partir de dos fuentes inéditas localizadas en el Archivio di Stato di Firenze y del Archivo Histórico de Protocolos de Granada. En primer lugar analizaremos la importación de espadas a través del puerto de Livorno, puerto de Florencia y la Toscana, desde los puertos de Cádiz y Alicante, entre otros. En segundo lugar nos centraremos en la importación de espadas italianas, que llevaron a cabo en Granada ciertas compañías comerciales genovesas. Estos mercaderes abastecieron a los espaderos granadinos — unos grandes desconocidos por la historiografía— de "hojas de espada" italianas de Milán, Brescia, Serravalle y Génova.

This paper analyses sword trade between Spain and Italy in sixteenth and seventeenth centuries through the study of two commercial routes used by European commercial networks from two unpublished sources: Archivio di Stato di Firenze and Archivo Historico de Protocolos de Granada. Firstly, we will examine sword importation through Leghorn, port of Florence and Tuscany, from the Spanish ports of Cadiz and Alicante, among others. Secondly, we will analyse Italian sword trade carried out by Genoese commercial companies placed in Granada. These merchants supplied swordsmiths from Granada - almost unknown by historiography — with Italian sword blades from Milan, Brescia, Serravalle and Genoa.

\section{Palabras Clave - Keywords}

Espadas; Comercio; redes de mercaderes; Italia; España; Siglos XVI y XVII; Florencia; Livorno; Alicante; Cádiz; Granada; Espaderos; Milán; Brescia; Serravalle; Europa.

Swords; Trade; merchant networks; Italy; Spain; sixteenth and seventeenth centuries; Florence; Leghorn; Alicante, Cadiz; Granada; Sword makers; Milan; Brescia: Serravalle; Europe.

\section{INTRODUCCIÓN Y ESTADO DE LA CUESTIÓN}

Rey: Si, respondedme la verdad de lo que deciros quiero. Coscorrón: ¿La verdad? Pregunte "tu” majestad. Rey: ¿Cómo la espada sacó quebrada?

1 Este artículo es uno de los resultados de la investigación realizada gracias a una beca postdoctoral 2014-2015 Mellon Fellowship en Villa I Tatti-The Harvard University Center for Italian Renaissance Studies de Florencia.

* Universidad de Granada, Departamento de Historia Moderna y de América, rgiron@ugr.es, http://orcid.org/00000003-1055-7729. 
Coscorrón: ¿Qué duda es esa? Era espada genovesa y de un alcance quebró.

Francisco de Rojas Zorrilla, No hay ser padre siendo rey (1635)

Lope: ¿Qué es aquesto?

Sancho: Nada: dejadme enderezar aquesta espada.

Lope: Que suspendáis vuestro valor me pesa.

Sancho: Tuércese fácilmente, es genovesa.

Lope: Acabad.

Sancho: Vive Dios, que un real no vale.

Francisco de Rojas Zorrilla, Donde hay agravios no hay celos (1640)

Las dos citas de Francisco de Rojas Zorrilla sobre las espadas genovesas nos introducen con humor a la existencia de espadas de origen italiano en la España de los Austrias, haciendo referencia a aquellas de menor precio y calidad: las que se forjaban en Génova. El uso de las espadas "civiles" o "de duelo" se popularizó mucho con la invención, a principios del siglo XVI, de una espada ligera, la espada ropera — rapier —, que dio lugar a nuevas ramas de la esgrima y que para su enseñanza contó con numerosos centros y exitosos tratados de su uso por toda Europa ${ }^{2}$, aunque no fue esta espada el único de los tipos producidos en España, pues también encontramos estoques, montantes y verdugos ${ }^{3}$.

Las nuevas espadas desbordaron el ámbito militar para convertirse en un elemento más de la indumentaria civil. En teoría, todo varón adulto, especialmente de las élites, debía po-

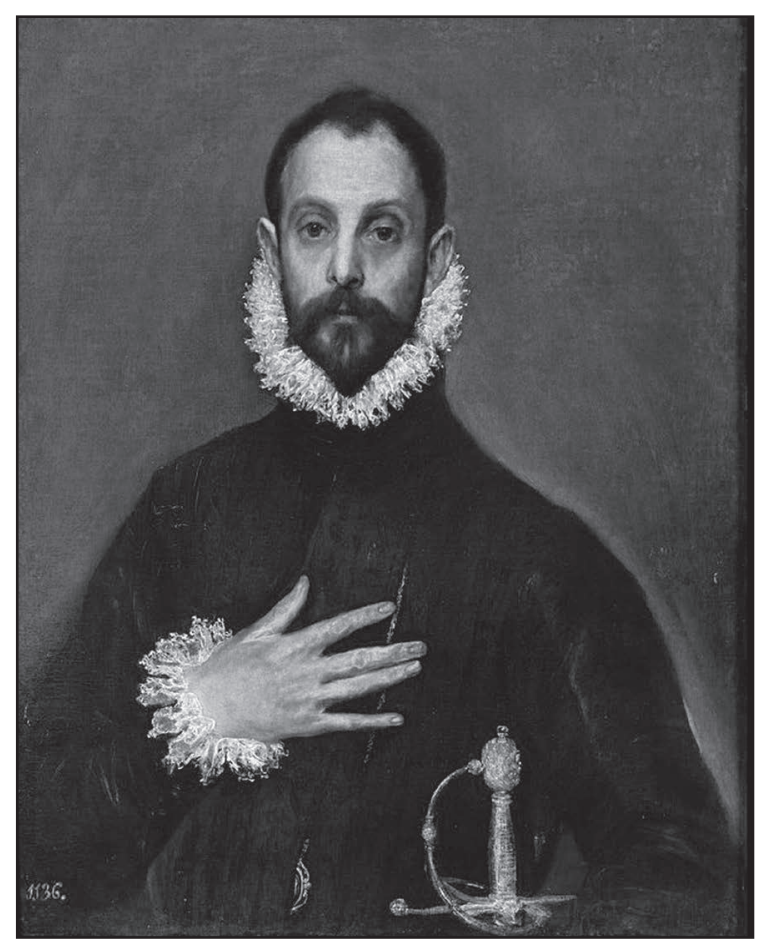

Figura 1. El Greco. El caballero de la mano en el pecho (1580). Museo del Prado, Madrid.

2 Norman, 1980

3 Dueñas Beraiz, 2004: 212. 
seer una espada, lo que seguramente supuso un enorme tirón para la industria armamentística europea. La asociación entre espada y nobleza se hace patente en la Historia del Arte, donde son raros los retratos masculinos sin espada - de la que generalmente vemos solo la empuñadura - ya que incluso los niños, especialmente de la realeza, aparecen portando este arma blanca.

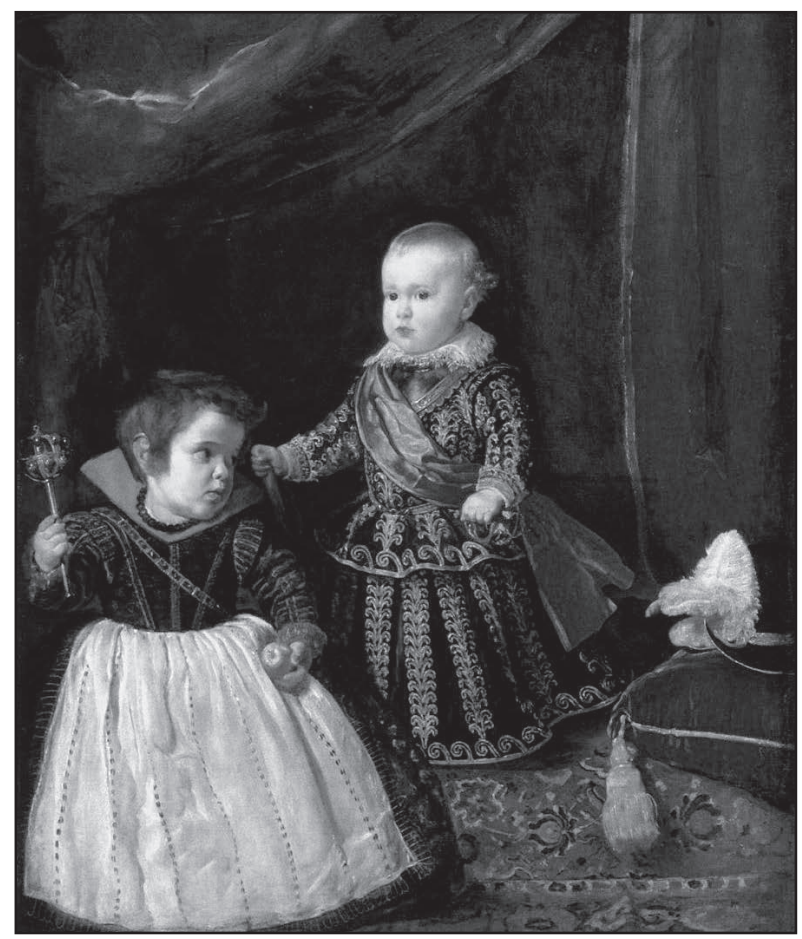

Figura 2. Diego Velázquez, El príncipe Baltasar Carlos con un enano (1632). Museum of Fine Arts, Boston.

La realidad, sorprendentemente, desborda la teoría, ya que en la práctica todo varón adulto - noble o no- pudo ser portador de una espada en la Edad Moderna. Así quedaba patente en un memorial enviado al rey Felipe II a finales del siglo XVI, cuando se realizó un inventario de las armas - arcabuces, ballestas, picas, morriones - de los quince pueblos del partido de Quintanar en La Mancha, señalando que

...no irán puestas las armas menudas como son espadas, rodelas, lanzas comunes y otras armas semejantes sino solamente las que más hacen al caso, porque las espadas, se ha de entender, que cada vecino de los que irán declarados que hay en cada pueblo tiene una espada y algunas otras armas comunes ${ }^{4}$.

Si el ejemplo de Quintanar se extrapola al resto del territorio castellano y aragonés, cada uno de los varones adultos de la España de los Habsburgo poseía una espada, por lo que millo-

4 López Salazar Pérez, 2005: 54. 
nes de ellas formaron parte de la cotidianidad en dichos reinos y en otros territorios europeos. ¿Podríamos hablar de una democratización del uso de la espada a finales del siglo XVI?

Numerosos centros de producción a lo largo y ancho de Europa se especializaron en las espadas citadas, destacando las producciones italianas, alemanas y españolas. En el caso español, el acero vasco producido en Mondragón ${ }^{5}$ o aceros importados de diferentes regiones europeas, fueron templados por expertos espaderos de las ciudades de Toledo ${ }^{6}$, Bilbao ${ }^{7}$, Barcelona, Valencia, Zaragoza ${ }^{8}$ y Sevilla ${ }^{9}$, entre otras ${ }^{10}$. En los inventarios de las armerías reales o de los miembros de la alta nobleza castellana encontramos espadas de todas estas procedencias ${ }^{11}$ si bien, por encima de todas ellas, destacaba la ciudad de Toledo dando espaderos como los Sahagún, cuyas espadas hoy en día - a la manera de los violines Stradivarius o Guarneriusson el objeto más deseado de los coleccionistas de armas blancas, que ya en su época recibían elogios como este de Lope de Vega de la comedia Las flores de don Juan ${ }^{12}$

Espadero: ¡Vive Dios que es un diamante!

Don Alonso: Aún diamante es común

que espada de Sahagún

no ha de tener semejante.

Fuera de España encontramos las famosas y demandadas espadas de Solingen - acaso el mayor centro de producción europeo- y Passau ambas en Alemania ${ }^{13}$; Milán y Brescia (Italia); Toulouse, Reims y Tours (Francia); Lisboa ${ }^{14}$ (Portugal) entre otros muchos centros ${ }^{15}$. También merecieron fama en el mundo musulmán las espadas de Damasco y Fez ${ }^{16}$. Todo lo anterior hace pensar que las espadas, o más concretamente las hojas de espadas, fueron objeto de un incesante comercio entre estos centros y las ciudades que las demandaban en el Viejo y Nuevo Mundo. Todas estas calidades y procedencias se veían reflejadas en su precio. Contamos con dos pragmáticas para el siglo XVII que nos van a informar de ello: una de 1627 y otra de 1680 donde se fijaban los precios máximos para las espadas:

Tabla 1: Precios máximos de hojas de espadas fijadas por Pragmáticas según su procedencia (s. XVII) ${ }^{17}$

\begin{tabular}{|l|c|c|}
\hline Origen & \multicolumn{2}{|c|}{ Precios (en reales) según el año 1627 1680 } \\
\hline Toledo & 24 & 30 \\
\hline Alemania & 13 & 18 \\
\hline Toulouse y Francia & 11 & 11 \\
\hline Génova & 9 & 10 \\
\hline
\end{tabular}

5 Carrión Arregui, 1998: 29. Larrañaga, 1984 y 1988. Azpiazu, 1994.

6 Leguina, 1885, 1897, 1912; Bernadet, 1891; Peláez Valle, 1983 y 1986; Dueñas Beraiz, 2003.

7 Larrañaga, 1981; Dueñas Beraiz, 2001.

8 Dueñas Beraiz, 2000.

9 Dueñas Beraiz, 2004: 251-256.

10 Enrique de Leguina (1912: 363) completa la nómina con San Clemente (Cuenca), Madrid, Cuenca, Villalón, Cuéllar, Badajoz, Segovia, Valladolid, Calatayud, Orgaz, Mondragón, Ávila, Alcázar de San Juan, Albacete, Chinchilla, El Bonillo, Aspe, Baeza, Jaén, Sigüenza, Guadix, Murcia, Navarra, Galicia y Granada.

11 Cano de Gardoqui, 2000; González Ramos, 2014.

12 Peláez Valle, 1983: 170.

13 Wagner, 1969: 234-298

14 Mark y Silva Horta, 2011: 103-134.

15 Norman, 1980 y Leguina, 1912: 363

16 Leguina, 1912: 361.

17 Fuente: Viñas Mey, 1968: 739 y Peláez Valle, 1983: 168. Elaboración propia. 
En el mercado castellano, la primacía de la espada toledana quedaba perfectamente reflejada en su precio, siendo seguida por las espadas alemanas, francesas y, por último, — como vimos en las citas del comienzo- las genovesas. Entre las dos pragmáticas hay un notable ascenso del precio de las espadas toledanas y alemanas - algo esperable si se tiene en cuenta la "revolución de los precios" por la inflación - mientras que las francesas y genovesas mantienen su precio prácticamente inalterado. En cualquier caso, las espadas se nos presentan asequibles a gran parte de la población, por lo que casi cualquier vecino de Castilla pudo adquirir una, al menos, de las baratas de Génova y Francia.

Este comercio, sin embargo, no ha suscitado hasta la fecha demasiado interés en los investigadores. Si bien hay artículos donde al tratar un tipo de espada o la producción de una determinada ciudad se hacen breves menciones a su comercio, no contamos con artículos específicos sobre las redes comerciales o el comercio de espadas propiamente dicho. Así, para el caso español, se sabe que espadas de Bilbao se exportaron a América, Irlanda, o Inglaterra en donde eran conocidas como Bilbo ${ }^{18}$. En este sentido el The Marchants Avizo de 1589 informaba a los mercaderes ingleses que en los puertos de San Sebastián y Bilbao se podrían encontrar hierro y "hojas de espadas" entre otras mercancías ${ }^{19}$.

Desde el enfoque de la Historia del Arte o de la museística, conocemos que espadas toledanas terminaron en Italia y hoy llenan, por ejemplo, algunos museos florentinos como el Stibbert Museum o la Armeria medicea. Es lógico pensar que algunas espadas llegaron en forma de regalos entre las cortes de los Austria o los Medici, de la misma forma que llegaron objetos de $\operatorname{arte}^{20}$; pero la demanda de la élite florentina y de los militares españoles sobre el terreno, especialmente de estos objetos de uso cotidiano, plantea la necesidad de otras vías. ¿Cómo llegaron a Italia estas espadas? ¿Llegaron a otros países europeos o al Nuevo Mundo?

La particular condición de las espadas como producto estratégico — al tratarse de armas y tener un uso militar, hacía necesario obtener una licencia para sacarlas al exterior- justifica que no en pocas ocasiones aparezcan espadas y hojas de espada entre los objetos incluidos en las llamadas "cédulas de paso". En un trabajo de Alfredo Alvar para finales del siglo XVI encontramos a diversos militares y cortesanos llevando estas armas en su viaje a Flandes. Así en 1576 el Correo del Rey, Juan de la Porte, llevó entre sus posesiones "doce hojas de espada"; el organista de Felipe II, Miguel de Boveque, seis; el arquero Cornelio de Achere, tres hojas; y el arquero real Juan de la Haya llevó una ${ }^{21}$. Tres años después el coronel Cristóbal de Mondragón llevaba varias espadas entre sus objetos personales ${ }^{22}$.

Lo mismo ocurrió cuando particulares quisieron llevar espadas y otras armas a Indias. Varios fondos de las Audiencias novohispanas dentro del Archivo General de Indias cuentan con estas "licencias de armas" vinculadas a peticiones de particulares al Consejo de Indias. Entre los muchos casos traeremos aquí el de Juan Sedano que en 1554 quería pasar al Perú con "dos coracinas con sus faldas y mangas de mallas, tres cotas de mallas, tres celadas, tres cascos, seis arcabuces, cuatro alabardas, un arnés, dos ballestas, seis lanzas, una docena de espadas, cuatro rodelas y media docena de dagas" ${ }^{23}$. Desgraciadamente, tanto en el caso de las "cédulas de paso" como en el de las "licencias de armas" a Indias, no aparece el origen o la calidad de las espadas, si bien pueden ser una pista a seguir.

18 Dueñas Beraiz, 2001: 272-275;

19 Lanero Fernández, 2008: 160.

Goldberg, 1996.

1 Alvar Ezquerra, 1996: 108.

Ídem: 102.

Archivo General de Indias, Lima, 567, L. 7, f. 491r 
Sevilla se nos presenta como un lugar clave para la distribución de las espadas forjadas con acero vasco. Importantes compañías comerciales especializadas en todo tipo de materiales metálicos abastecieron las Indias de armas y material bélico variado ${ }^{24}$. María del Carmen Mena García publica la llegada en una fecha tan temprana como 1513 de un gran envío de armas blancas procedentes de Bilbao y Durango entre las que se encontraban 197 espadas "con sus vainas guarnecidas" así como todo tipo de lanzas y picas ${ }^{25}$. José Antonio Azpiazu documenta envíos a esta ciudad de grandes cantidades de arcabuces y picas, pero no, curiosamente, de espadas encargadas por mercaderes vascos avecindados en Sevilla a finales del siglo XVI ${ }^{26}$. Por nuestra parte hemos localizado a una de estas compañías, la de los vascos Martín de Arregui, Francisco de Aguirre y Dionisio Adame de Arreguia oriundos de Placencia de las Armas - Soraluce - que operó en la ciudad andaluza al menos entre 1586 y 1596. En un inventario de 1593 de su almacén de Sevilla aparecen entre sus mercancías un buen número de armas y armaduras entre las que encontramos 18 hojas de espada ${ }^{27}$.

Tabla 2: Armas, armaduras y material armamentístico de Martín de Arregui \& Cía en Sevilla $(1593)^{28}$

\begin{tabular}{|l|c|c|}
\hline Producto & Cantidad & Precio/unidad (mrs) \\
\hline Escopetas & 56 & 800 \\
\hline Cañones de escopetas & 11 & 500 \\
\hline Arcabuces sin aderezos & 264 & 612 \\
\hline Mosquetes sin aderezos & 129 & 1.496 \\
\hline Cuerpos de armas & 30 & 800 \\
\hline Morriones & 60 & 200 \\
\hline Espadas & 18 & 200 \\
\hline Hachas pequeñas y medianas aceradas & 545 & 119 \\
\hline Hachas aceradas grandes & 323 & 136 \\
\hline Hachas baladíes grandes & 407 & 68 \\
\hline Hachas medianas y pequeñas & 499 & 42 \\
\hline Frascos de arcabuces & 339 & 51 \\
\hline
\end{tabular}

Este comercio continuó en los siglos siguientes. Fernando Fernández cita a los mercaderes Pedro de Urrutia y Juan Antonio Zulueta Aragón fletando a Indias varios cajones de espadas cada uno, en la segunda mitad del siglo XVII ${ }^{29}$. Sevilla no fue el único destino de las espadas vascas. Hilario Casado al estudiar los seguros burgaleses a finales del siglo XVI encuentra envíos de hojas de espadas y otras armas a Lisboa u Oporto ${ }^{30}$.

¿Ocurrió lo mismo con las espadas italianas en España? Como ya vimos en la cita de Rojas y en las pragmáticas, el acero italiano se comercializó profusamente en España. Gracias a los artículos de Hilario Rodríguez de Gracia, dedicados a los mercaderes genoveses en Toledo,

\footnotetext{
24 Azpiazu, 1994.

25 Mena García, 1998.

26 Azpiazu, 1994: 44.

27 Archivo Histórico Provincial de Sevilla, PNS, 12.569, f. 523v.

28 Fuente: Ídem, f. 520v y ss. Elaboración propia.

29 Fernández González, 2000: 353-354.

30 Casado Alonso, 2003.
} 
tenemos algunas noticias de que hojas de espadas italianas fueron importadas a finales del siglo XVI por los citados mercaderes, suministrando a los espaderos toledanos — junto a los mercaderes vascos del famoso acero helado- la materia prima para sus famosas forjas ${ }^{31}$. La documentación nos dice que las hojas de espadas provenían de las herrerías de Génova o Milán, y la historiografía asevera que en Toledo se templaban y añadían empuñaduras junto con otras guarniciones.

Tabla 3. Ejemplos de importaciones de hojas de espadas italianas en Toledo (1587-1621) $)^{32}$

\begin{tabular}{|l|c|l|l|l|c|}
\hline Año & Número & Origen & Compañía & Comprador & $\begin{array}{c}\text { Precio } \\
\text { (reales/hoja) }\end{array}$ \\
\hline 1587 & 338 & Génova & Ansaldo & Pedro Hernández, espadero & - \\
\hline 1590 & 360 & Génova & Bocangelino y Baba & Pedro Hernández, espadero & 4 \\
\hline 1593 & 250 & Milán & Juan Bautista Dosio & Lorenzo de la Fuente, espadero & - \\
\hline 1621 & 200 & Génova & $\begin{array}{l}\text { Esteban Cebolín y } \\
\text { Antonio Dungo }\end{array}$ & Francisco Díaz, espadero & 4.5 \\
\hline
\end{tabular}

Igualmente, en el Levante español se tiene constancia de la llegada de espadas italianas. Por ejemplo, en las noticias del naufragio de la nave La Marolina en las costas de Denia, en el reino de Valencia en 1571, los mercaderes consiguieron rescatar un envío de hojas de espadas. El barco había salido de Génova por lo que imaginamos se trataban de espadas italianas, bien de la citada ciudad o de Milán ${ }^{33}$. Unos años después, en 1597, el mercader galo Joan Augier importó hojas de espadas genovesas y arcabuces ${ }^{34}$ y los italianos Cernesio en 1615 tenían en su poder hojas de espadas llegadas de Mallorca pero seguramente de procedencia italiana ${ }^{35}$.

En el sur peninsular también quedan ejemplos de este comercio. En Sevilla llegaron desde Génova numerosas remesas de espadas. Enrique Otte para principios del siglo XVI da algunos ejemplos. En 1501 el genovés Battista Cattaneo vendía a su compatriota Benito Vayrola, cuchillero, 36 hojas de espadas por $2.160 \mathrm{mrs}$ y en 1515 el concurso de acreedores del genovés Bernardo Pinello arrojaba buen número de espadas italianas entre las mercancías a subastar ${ }^{36}$. Otte llega a afirmar que los genoveses poseían el monopolio del comercio de espadas y otras manufacturas de acero en la Sevilla de principios del siglo $\mathrm{XVI}^{37}$. A finales de este siglo sabemos que el mercader vasco residente en Sevilla, Lope de Ugarte, contaba entre sus bienes "una espada y daga con guarniciones de Italia labrada" y "otra espada y daga con sus guarniciones". Ambas armas fueron subastadas a su muerte por su albacea Juan de Munibe por 44 y 29 reales respectivamente ${ }^{38}$. Unos precios muy altos que nos hace pensar que tal vez se trataba de espadas "mixtas" con hojas toledanas ensambladas en Italia o bien espadas italianas de calidad superior a la comercial. Finalmente, en 1598 encontramos referencias a un cargamento legado a Sevilla y con posible destino a la ciudad de Córdoba de "dos balas de hojas de espadas de

31 Rodríguez de Gracia, 2005 b: 179 nota 77.

2 Fuente: Rodríguez de Gracia, 2005 a: 610 nota 70 y Rodríguez de Gracia, 2005 b: 179 nota 77 . Elaboración propia.

3 Muñoz Navarro, 2010: 326.

Pérez Villalba, 2012: 340.

San Ruperto Albert, 2013: 5.

Otte, 1996: 157 y 166.

Ídem, 157.

AHPSe, PNS, 12.568 , ff. 302r y $767 \mathrm{r}$ 
Génova" con un valor de 1.452 reales, lo que arroja una cifra exacta de 363 espadas genovesas a cuatro reales cada una ${ }^{39}$.

También entraron en el mercado castellano espadas italianas por el puerto de Málaga. Allí los genoveses Italián y Centurión vendieron 20 espadas de origen genovés en una fecha tan temprana como $1496^{40}$; pero, el caso más espectacular alude a las 5.400 hojas de espadas, que importaron en 1626 los genoveses Bartolomé Baldasano y Juan Agustín Capelo a través del puerto de Málaga, siguiendo el trabajo de Vicente Montojo ${ }^{41}$. Aunque desconocemos el origen de las mismas, no sería desacertado pensar en un origen genovés.

Pero me atrevo a decir que fue por los puertos de Alicante y Cartagena por donde los mercaderes genoveses importaron el mayor número de espadas italianas. Francisco Velasco se refiere a la llegada en 1591 de " 44 docenas de espadas y 8 espadines" — 536 piezas — procedentes de Italia con un valor de 2.233 reales $^{42}$. Por estos puertos los mercaderes genoveses de Granada importaron espadas italianas como yo mismo indiqué en un epígrafe de mi tesis doctoral, información que veremos ampliada en el tercer apartado de este artículo ${ }^{43}$.

Las espadas italianas no fueron el único acero transpirenaico que se comercializó en España, como veíamos en los precios y procedencias de las premáticas de 1627 y 1680 . El intenso comercio con los puertos de los Países Bajos y Francia a finales del siglo XVI y en los años siguientes, pese a tratarse de naciones casi siempre enemigas de la Monarquía Hispánica, permitió la llegada al mercado castellano de espadas de procedencia Atlántica, especialmente hojas francesas, holandesas y alemanas. Manuel Lobo en su estudio sobre el comercio canario nos dice que espadas francesas y holandesas llegaron a las Islas a finales del siglo XVI ${ }^{44}$; Vicente Montojo se refiere a una caja de espadas procedente de Ámsterdam, transportada a Alicante por cuenta de Francisco Langenhort y Bautista Van Halen a finales del siglo XVII ${ }^{45}$; y Jesús Torrealba cita a un maestro espadero de Logroño, Andrés Ruiz de Villalba, que en 1690 tenía en su poder 200 hojas de espadas de Alemania junto con 20 hojas "ordinarias" forjadas en Tolosa $^{46}$. Por último nos planteamos cuántas espadas de estas procedencias entraron en España por los cauces ilegales, por el contrabando. Ángel Alloza refleja en uno de sus trabajos como espadas, entre otras manufacturas metálicas, fabricadas por países enemigos de la Monarquía llegaron a bordo de navíos procedentes de ciudades de la Hansa en las primeras décadas del siglo XVII. Las espadas confiscadas eran subastadas en la Junta del Almirantazgo por valores muy inferiores a su precio en el mercado ${ }^{47}$. Escasos ejemplos para un comercio - legal o ilegal - que intuimos alcanzó altas cotas de intercambio.

Vemos, por tanto, que el comercio de espadas atiende a dinámicas complejas y variadas, donde varios centros de producción de hierro y acero suministraban la materia prima para las armerías más conocidas, gracias a numerosas redes comerciales que interactuaban entre ellas y cuyo estudio general escapa a las expectativas de este artículo. No obstante, creemos que podemos aportar cierta luz a lo que al comercio entre Italia y España se refiere. Este comercio se define por la existencia de dos flujos opuestos pero complementarios: hojas de espadas espa-

39 AHPSe, PNS, 9.302, f. 788r. Agradezco esta referencia al profesor de la Universidad de Sevilla Manuel Fernández Chaves.

40 López de Coca y López Beltrán, 1980: 110.

41 Montojo Montojo, 1993: 330 nota 1406. Agradezco a Vicente Montojo esta referencia.

42 Velasco Hernández, 2001: 250.

43 Girón Pascual, 2013: 192-193.

44 Lobo Cabrera, 2015: 354. Los inventarios notariales post-mortem también confirman la llegada de espadas toledanas a las Islas. Agradezco al profesor Lobo, esta referencia.

45 Montojo Montojo, 2011: 334.

46 Torrealba Domínguez, 1992: 101.

47 Alloza Aparicio, 2003: 227 y 242. 
ñolas que se exportaban a Italia — entendemos que de buena calidad - y hojas de espadas italianas que se importaban hacia España, siendo su calidad y precio inferiores. Una vez llegadas a su destino, los espaderos locales las templaban y adaptaban a los gustos y modas de su tierra.

Este artículo va a analizar estos dos flujos comerciales por medio del estudio de documentación inédita conservada en el Archivo di Stato di Firenze (Florencia) y en el Archivo Histórico de Protocolos de Granada. El archivo florentino nos va a permitir situar la llegada intermitente de espadas de procedencia española al puerto de Livorno, entre 1568 y 1600 y las redes comerciales tanto castellanas como florentinas que realizaron dicho comercio. El archivo granadino nos va a ayudar, paralelamente, a constatar la llegada de espadas italianas - Milán, Serravalle (Treviso) y Brescia - por medio de redes comerciales genovesas, al mismo tiempo que nos descubren sus clientes, los espaderos de Granada, bastante numerosos y grandes desconocidos de la historiografía.

\section{LIVORNO COMO PUERTO DE ENTRADA DE ESPADAS ESPAÑOLAS EN ITALIA}

A finales del siglo XVI, poderosas y activas redes comerciales de mercaderes florentinos, genoveses y castellanos controlaban el comercio entre los puertos españoles y Livorno ${ }^{48}$. Estas redes traficaban con una gran variedad de productos tanto de origen peninsular como de las Indias de Castilla. El producto principal —o desde luego más importante en volumen - fue la lana castellana transportada en carros desde todos los reinos peninsulares hasta Alicante, principal puerto en el comercio entre España e Italia de la época. Junto a ella salieron otros productos fundamentales para la economía de la Toscana como la cochinilla de Nueva España, tinte rojo y clave para los ricos paños y sedas florentinos — sobre todo las rascias o rajas-; los cueros de Indias, o el azúcar, indispensable como conservante de la notable producción orto-frutícola de la región, se unían a otros alimentos como el atún, la pasa, el aceite de oliva — también usado en la industria pañera - o el turrón de Alicante; joyas sudamericanas esmeraldas, perlas —; medicinas — palo guayaco, zarzaparrilla, jengibre; especias — canela, azafrán, pimienta, clavos "de comer"- y manufacturas castellanas como los guadameciles, cordobanes y las sedas granadinas.

Estas mismas redes fueron las que comerciaron con espadas. Las portate navi del Archivio di Stato di Firenze nos informan de la llegada al puerto de Livorno de envíos de espadas spade - y hojas de espadas - lame di spade — desde los puertos de Alicante y Cádiz, entre otros. Se han revisado unos 700 registros de navíos entre los puertos españoles y Livorno en el periodo 1568-1600, apareciendo 25 referencias de envíos de espadas y hojas de espadas.

No podemos concretar el número total, ya que la documentación nos viene expresada en cajas, haces, y todo tipo de paquetes: involto, rivolto, fangotto, fardello...donde muy raramente nos dan una cifra y si lo dan es casi siempre para cantidades pequeñas — de tres a seis - espadas terminadas. No obstante la forma de las hojas de espadas facilita su transporte pudiendo apilarse en una caja o un fardo varias docenas de ellas.

48 Braudel y Romano, 1951; Ruiz Martín, 1965; Filippini, 1998; Ghezzi, 2007. 
Tabla 4. Envíos de espadas desde puertos españoles al puerto de Livorno (1573-1592) ${ }^{49}$

\begin{tabular}{|c|c|c|c|}
\hline Fecha & Puerto de origen & Cantidad "de spade" & Mercader/Consignatario \\
\hline 22-VIII-1573 & Alicante & 1 balleta & Piero Canigiani \\
\hline 13-IX-1573 & Alicante & 1 fascio & Baltasar Suárez \\
\hline 13-III-1574 & Alicante & 1 fardello & Batista Arrighi \\
\hline $29-X-1577$ & Alicante & 1 casseta & Batista Ricasoli \\
\hline 9-VIII-1578 & Alicante y Valencia & 5 fardelli & Franco Marello \\
\hline $1-X I-1578$ & Alicante & 1 casseta & Donofrio de Milán \\
\hline 9-II-1580 & Alicante & 1 fangotto & Campana\&Coletta \\
\hline 15 -III-1580 & Cádiz & 3 "rivolti" & Gio B. Altoviti \\
\hline 31-III-1580 & Sevilla & 5 espadas & A quien las espere \\
\hline 18-VIII-1580 & Alicante & 6 "lame di spade" & Capponi \\
\hline $23-I-1581$ & Alicante & 1 fascio & Jacopo Scotti \\
\hline $25-I V-1581$ & Cádiz, Cartagena, Alicante & 1 cassa & Nicolo Bartoli \\
\hline 28-XII-1583 & Alicante & 1 fangotto & Campani \\
\hline 14-VIII-1584 & Barcelona & 3 espadas & Ettore Buonacorsi \\
\hline 30-III-1586 & Cádiz & 1 involto & Jacopo Ricciardini \\
\hline $31-\mathrm{III}-1586$ & Cádiz & 1 fardello & Gio B. Ricasoli \\
\hline $12-\mathrm{V}-1587$ & Lisboa y Cádiz & 1 involto & Neri \\
\hline $12-\mathrm{V}-1587$ & Lisboa y Cádiz & 1 involto & Filippo Ricasoli \\
\hline $12-V-1589$ & Lisboa y Cádiz & 1 "rivolto" & Neretti \\
\hline $12-V-1589$ & Lisboa y Cádiz & 1 fangotto & Ricasoli \\
\hline $8-V I-1590$ & Cádiz y Alicante & 1 fardo & Berti \\
\hline 8 -III-1592 & Cádiz & 1 fangotto & Antonio Salinas \\
\hline 23-VIII-1592 & Cádiz y Alicante & 3 involti & Ettore Buonacorsi \\
\hline 23-VIII-1592 & Cádiz y Alicante & 1 "rivolto" & Becchuti \& Benti \\
\hline 23-VIII-1592 & Cádiz y Alicante & 2 casseta & Vittorio Satamanche \\
\hline
\end{tabular}

Cabe pensar que estos envíos serían similares a los que citaba Rodríguez de Gracia para el caso toledano, y por tanto contar con cantidades cercanas a la centena de espadas en cada uno de estos envíos.

A partir de la documentación florentina no podemos concretar el origen de las espadas que salieron desde los puertos españoles. Sería necesario tratar de localizar estos envíos en los registros de salida del puerto de Alicante o Cádiz, puertos que no cuentan con una documentación tan temprana. La otra opción sería tratar de encontrar los fletes de navíos en los protocolos notariales de ambas poblaciones, algo que para el caso de Alicante no es posible, pero parece que sí lo es para Cádiz o Sevilla. Así mismo los protocolos notariales florentinos pueden darnos pistas del origen de las espadas si localizamos a los mercaderes que las adquirieron.

Alicante en estas fechas centraliza buena parte del comercio mediterráneo de los reinos peninsulares, siendo el puerto principal de levante, por delante de Valencia o Barcelona. El comercio interior por carros de bueyes comunicaba el citado puerto con el interior de Castilla, por lo que parece plausible un origen toledano de buena parte de estas espadas. Cádiz y Sevilla estaban bien abastecidos de manufacturas de los Países Bajos o el Imperio pero también de los puertos cantábricos, por lo que el origen de dichas armas bien podría ser las forjas vascas

49 Fuente: Archivio di Stato di Firenze, Mediceo del Principato, 2079, 2080, 2139. Elaboración propia. 
o las famosas espadas germánicas de Solingen o Passau. Lisboa también aparece como puerto de salida de uno de los barcos y que llegan a Livorno con cuatro cargas de espadas. Ya vimos que Hilario Casado localiza llegadas de espadas vascas a este puerto y por el libro de Mark y Silva Horta sabemos que el puerto luso era otro centro de ensamblaje de espadas a partir de hojas italianas y flamencas, con destino a los mercados coloniales portugueses de la África subsahariana, donde eran intercambiadas por esclavos por mercaderes de origen judeoconver$\mathrm{so}^{50}$. Lisboa también recibía espadas de origen indio, muy valiosas y exóticas; tal vez algunas de estas fueron redirigidas a Livorno.

Podemos analizar con mayor detenimiento las redes comerciales receptoras de las espadas. Los dos castellanos que actuaron como consignatarios de dos lotes, Antonio de Salinas y Baltasar Suárez nos son bien conocidos. El primero era el agente de numerosos mercaderes y ganaderos burgaleses y el segundo el representante de los ganaderos segovianos que exportaban la mejor lana merina a Italia. Tanto el uno como el otro tenían contactos en Sevilla y otras plazas comerciales castellanas como Medina del Campo, entre los que encontramos a Simón Ruiz, que también los tenía en Francia ${ }^{51}$. Debido al carácter internacional de estos mercaderes no nos atrevemos a adivinar el origen de las espadas, sin realizar estudios más específicos.

El resto de los mercaderes son todos florentinos. Destacan los poderosos Capponi y Neretti banqueros florentinos, con espléndidas relaciones con Felipe II y asentistas suyos. Neri es socio de los Fantoni, muy involucrados en el comercio con Indias a través del puerto de Cádiz. Ricasoli y Buonacorsi, que son los que más lotes reciben, aparecen en la documentación como receptores de mercancías, de lugares tan distantes como Ámsterdam, Alejandría o Berbería por lo que no podemos afinar más. Como hemos dicho, necesitamos "tirar del hilo" en las fuentes locales para conocer el origen de las espadas, o colocar la "primera piedra" para que otros investigadores continúen la investigación aquí iniciada.

\section{LOS MERCADERES GENOVESES, LAS ESPADAS ITALIANAS Y EL MERCADO GRANADINO}

Como hemos visto en el apartado anterior el comercio de espadas entre España e Italia suministró aceros españoles - y puede que de la Europa Atlántica - a la corte Medicea - y al resto de Italia - a través del puerto de Livorno. Pero este flujo "se cruzó" con otro opuesto que enlazaba el norte de Italia con los puertos levantinos de Alicante y Cartagena y, en menor medida con los de Málaga y Sevilla. A través de los primeros y tras un largo transporte a lomos de bueyes, la ciudad de Granada se abasteció de numerosos productos itálicos como el papel, los espejos, paños y telas de Florencia y Venecia, oro hilado y guantes de Milán, manufacturas y $\operatorname{armas}^{52}$.

Este comercio estuvo monopolizado por mercaderes genoveses durante los siglos XVI y XVII. Se trataba de grandes compañías comerciales forjadas en torno a familias o clanes - los Mayolo, los Veneroso, los Escalla, los Cibo, los Invrea, los Nasso, entre muchos otros- que tejieron redes comerciales y políticas con las élites locales, llegando a controlar las principales producciones del reino, como la lana y el azúcar, que a su vez exportaban a Génova y Livor$n^{53}$. Si bien esta participación data de época Nazarí hay un "boom" genovés en torno a 1580 , cuando aumenta el número de compañías y su riqueza, casi todas provenientes de miembros de

\footnotetext{
50 Mark y Silva Horta, 2011: 123.

1 Ruiz Martín, 1965.

2 Girón, 2013: 167.

3 Ídem: 37-46.
} 
la nobleza nueva genovesa. A partir de la documentación notarial hemos encontrado algunos ejemplos que pueden resultar de interés para analizar la llegada de espadas italianas a través de estas redes y compañías genovesas.

Los compradores de estas espadas van a ser en su mayoría espaderos locales. Sorprende que entre las ciudades productoras de espadas en España no se incluya a Granada, con una notable tradición nazarí en la fabricación de armas blancas ${ }^{54}$ y con ciertas técnicas como el adamascado y las originales tipologías de las espadas jinetas - llamadas también espadas moriscas - o de las dagas de orejas, teniendo estas últimas un notable éxito en Europa ${ }^{55}$; siendo además las ordenanzas de espaderos granadinas de 1531, una de las mejor conocidas y estudiadas ${ }^{56}$. Leyendas en torno a los espaderos granadinos como la de Julián del Rey ${ }^{57}$ respaldan la importancia de Granada como lugar de producción de espadas, pero muy poco sabemos de esta ocupación en la actualidad. No obstante, estos artesanos aparecen en la documentación granadina en un número apreciable, por eso nos sorprende que en el famoso censo de 1561 no aparezca ninguno de ellos, algo que contrasta con la documentación notarial y parroquial. ${ }^{58}$. La mayoría debió residir en la parroquia de la Magdalena, no lejos de la calle conocida como Horno espadero. Un trabajo manuscrito del siglo XIX de Bonifacio María Riaño Montero recopila referencias a los mismos en los libros parroquiales — partidas de matrimonio y bautismo - de la iglesia de dicha parroquia durante el siglo XVI ${ }^{59}$. Como era usual entre los artesanos la endogamia profesional es abrumadora: los matrimonios entre espaderos e hijas de espaderos se da en la mayoría de los casos, al igual que los padrinazgos entre los hijos e hijas de los espaderos y los colegas de oficio de sus padres. La nómina de espaderos recogida en dicho manuscrito y completada con las referencias que veremos a continuación, se recoge en el anexo documental. Pero veamos las numerosas transacciones que se "fraguaron" entre espaderos granadinos y mercaderes genoveses.

Durante la Guerra de las Alpujarras (1568-1570) los mercaderes genoveses se afanaron en suministrar materiales de guerra a los espaderos y otros artesanos granadinos. Un buen ejemplo de ello fueron las actuaciones del genovés Pelegro Mayolo en 1569. El 15 de enero concretaba la venta de "12 docenas de clavos de talabartes" con Pedro de Godina "el viejo", talabartero. Unos días después vendía dos arcabuces, dos tiros, una guarnición de espada, y una docena de talabartes al mercader granadino Pedro de Herrera ${ }^{60}$ y a final de mes hacía lo propio con 20 docenas de "talabartes de Milán" al talabartero Godina, ya citado ${ }^{61}$. A primeros de marzo hizo lo propio con Domingo Vázquez, guarnicionero, al venderle "doce docenas de bacines de talabartes" a 20 reales la docena ${ }^{62}$ y tres días después vendió a Jerónimo Ruiz, dorador, material bélico para equipar caballos - una docena de estribos, cuatro docenas y media de espuelas, once frenos de bridas, 29 pares de copas de brida para frenos - en las que se incluían "tres guarniciones de dagas" 63 . Del cuatro de marzo consta la primera escritura que tenemos entre relaciones comerciales entre mercaderes genoveses y espaderos granadinos. Mayolo se obligaba a entregar al espadero Diego Mexia "dos balas de hierro para puños de espadas" por un valor de 934 reales a pagar en ocho meses ${ }^{64}$.

\footnotetext{
54 Fernández Vega, 1935; Ferrandis Torres, 1943; Martínez Enamorado, 2005.

55 Sirva como ejemplo el éxito del espadero Diego de Zayas en la corte francesa. Blair, 1970.

56 Chancillería de Granada, 1672: 169-170.

57 Si bien el origen de este espadero no termina de estar claro. Dueñas Beraiz, 2000.

58 Ruiz Martín, 1968.

59 Biblioteca Central de Universidad de Granada BHR/Caja MS-2-047

60 Archivo Histórico de Protocolos de Granada [AHPG], G-168, s.f. (15-I-1569) y 39v.

61 Ídem, 54v

62 Ídem, $127 \mathrm{~V}$

63 Ídem, 131r

64 Ídem, 136r (4-III-1569)
} 
Estas relaciones comerciales no se limitaron a los convulsos años de la Guerra. Una década después, en 1582, encontramos a Pelegro Mayolo y su nueva compañía comercial con su sobrino Vicencio y su socio Juan Calvo Bernabo, entregando al espadero Pedro de Torres, que vivía en la céntrica Plaza de Bibarrambla "once docenas de hojas de espadas" a cuatro reales la hoja ${ }^{65}$. El bajo precio del material y la nacionalidad de los mercaderes nos hace pensar en una procedencia italiana; algo que se confirma unos meses después cuando los mismos actores realizan una transacción por 14 docenas "de espadas de Sarrabal o de Breza" por el mismo precio $^{66}$. Ahora queda claro el origen de las armas: se trataba de las poblaciones de Serravalle — que hemos localizado como un pequeño burgo de Vittorio-Venetto junto a Treviso- y Brescia, ambas en este tiempo bajo el control de la República de Venecia.

Las espadas de Serravalle tenían fama desde el siglo XIV y en la época contaban con algunos de los mejores armeros de Italia y la ciudad de Brescia - especialmente su anejo Gardone Val Trompia- desarrolló unas potentes manufacturas armamentísticas gracias a su fácil acceso a minas de hierro y carbón, que les permitió destacar al mismo tiempo como fabricantes de armas de fuego y blancas durante la Edad Moderna ${ }^{67}$. Desconocemos el mercader de armas intermediario que suministró las espadas a los Mayolo, pero no eran pocos los que se dedicaban a ello en estas poblaciones y periodo. No debe sorprendernos que las redes que suministraban los famosos espejos venecianos, muy numerosos en la documentación, hicieran lo mismo con las armas.

Una nueva compañía comercial aparece en 1588 traficando con armas de otra procedencia. Se trata de los hermanos Juan y Francisco Escalla, asimismo genoveses, que vendieron al espadero Luis de Aguilera "diez y seis docenas de hojas de espadas de Milán en prieto $^{68 "}$ a 50 reales la docena. El espadero pagaría la mitad de los 800 reales ese mismo mes, e iría pagando 24 reales cada sábado hasta abonar toda la deuda ${ }^{69}$. Estas 192 hojas de espadas de valor ligeramente superior a las de Brescia y Serravalle enlazan Granada — gracias a los mercaderes genoveses - con uno de los principales productores de armas de Europa: Milán ${ }^{70}$. De esta procedencia serán todas las espadas de las que hablaremos a continuación.

De nuevo en 1593 encontramos a los hermanos Escalla con otra partida de espadas milanesas, en esta ocasión para el espadero Salvador Barrero, morador del barrio de la Magdalena. El intermediario de los Escalla, Juan Bautista Rosso entregó 48 "espadas de Milán en prieto" a 46 reales la docena, pagaderos en cuatro meses ${ }^{71}$. Finalmente, en 1602, una tercera compañía ligur importa las citadas espadas. El genovés Pablo Vicencio Capriata venderá al regidor granadino don Fernando de Zapata y a Melchor de Almirante "el viejo", su fiador - y conocido mercader morisco- 673 "hojas de espadas de Milán" a 4 reales cada una". Se trata de una cantidad enorme y adquirida por un veinticuatro granadino, lo que nos sugiere que fuera una compra municipal para equipar varias compañías de la ciudad y destinarlas a los numerosos frentes que la Monarquía Hispánica tenía abiertos en estas fechas. Tan solo un mes después Capriata hará lo propio con el doctor Andrés Estacio, médico y vecino de Granada que abonó 850

65 AHPG, G-238, 580r (2-VII-1582)

66 Ídem, 1177r (26-XI-1582)

67 Barbiroli, 2012. De Brescia destacó la saga de maestros armeros iniciada por Bartolomeo Beretta que fundó en 1526 la compañía conocida en la actualidad por sus famosas pistolas. Sobre este asunto ver Belfanti, 1993 y 1998. Agradezco a Andrea Caracausi estas referencias.

68 Esta matización "en prieto" ¿puede hacer referencia a que las hojas de espada formaban una lámina de acero sin cortar?.

69 AHPG, G-271: 682r (11-V-1588)

70 Williams, 2012.

71 AHPG, G-303: 574r (31-V-1593)

72 AHPG, G-365: 1376v (4-X-1602) 
reales por 160 "hojas de espadas de Milán de un filo" a cinco reales y a pagar en ocho meses. Capriata traficaba con todo tipo de productos - azúcar, coral, espejos, drogas y especias- - y también con armas de fuego; en los registros del mercader Andrea Gandolfo se nos informa que éste había vendido por cuenta de Capriata "ciertas escopetas" valoradas en 700 reales ${ }^{73}$. Gandolfo hacía lo mismo con espadas valoradas en 212 reales enviadas por sus compatriotas y correspondientes en Sevilla, Rafael y Juan Ángel Tacio, no informándonos la documentación del origen de las dichas ${ }^{74}$.

Un último apunte indirecto sobre la importación de espadas data de 1604 donde el genovés Juan Esteban Chavarino autoriza al también mercader ligur Alejandro Fornari para cobrar de sus deudores ciertas cantidades. Curiosamente, todos menos uno - el genovés Juan Pablo Ayrolo- eran espaderos de la ciudad.

Tabla 5. Los espaderos granadinos deudores del mercader genovés Juan Esteban Chavarino en 1604 ${ }^{75}$

\begin{tabular}{|l|c|}
\hline Espadero & Deuda (reales) \\
\hline Justo Flores & 108 \\
\hline Francisco Pérez & 808 \\
\hline Juan Torres Colado & 808 \\
\hline Diego de Barreda & 808 \\
\hline Gaspar de los Reyes & 112 \\
\hline Luis García & 111 \\
\hline Salvador Barrero & 108 \\
\hline Nicolás de Fustamante & 108 \\
\hline Antonio Martínez & 108 \\
\hline Luis de Aguilera & 108 \\
\hline Pedro de Ávila & 108 \\
\hline
\end{tabular}

El poder remite a escrituras otorgadas el año anterior ante el escribano granadino Rodrigo de Rivera, documentación que desgraciadamente no se ha conservado en el Archivo. Puede llamar la atención que se repitan las cantidades, algo que suele responder a la división de lotes de mercancías entre diferentes compradores ${ }^{76}$. Podría tratarse, por tanto, de varios lotes de hojas de espadas — u otra mercancía análoga — que fue repartida entre los diferentes espaderos.

Tabla 6. Importaciones de hojas de espadas italianas en la ciudad de Granada (1582-1602) ${ }^{77}$

\begin{tabular}{|c|c|l|l|l|c|}
\hline Año & $\mathrm{N}^{\mathrm{o}}$ & Origen & Compañía & Comprador & $\begin{array}{c}\text { Precio } \\
\text { (reales/hoja) }\end{array}$ \\
\hline 1582 & 132 & $\begin{array}{l}\text { ¿Serravalle y } \\
\text { Brescia? }\end{array}$ & Pelegro Mayolo\&cia & Pedro Torres, espadero & 4 \\
\hline 1582 & 168 & Serravalle y Brescia & Pelegro Mayolo\&cia & Pedro Torres, espadero & 4 \\
\hline
\end{tabular}

73 AHPG, G-338: 43r-63r

74 Ídem

75 Fuente: AHPG, G-382: 95r. (30-I-1604). Elaboración propia.

76 Girón Pascual, 2013: 94.

77 Fuente: AHPG, G-271, G-365 


\begin{tabular}{|c|c|l|l|l|c|}
\hline Año & $\mathrm{N}^{\mathrm{o}}$ & Origen & Compañía & Comprador & $\begin{array}{c}\text { Precio } \\
\text { (reales/hoja) }\end{array}$ \\
\hline 1588 & 192 & Milán & $\begin{array}{l}\text { Juan y Francisco } \\
\text { Escalla }\end{array}$ & $\begin{array}{l}\text { Luis de Aguilera, } \\
\text { espadero }\end{array}$ & 4 \\
\hline 1593 & 48 & Milán & $\begin{array}{l}\text { Juan y Francisco } \\
\text { Escalla }\end{array}$ & $\begin{array}{l}\text { Salvador Barrero, } \\
\text { espadero }\end{array}$ & 4 \\
\hline 1602 & 673 & Milán & $\begin{array}{l}\text { Pablo Vicencio } \\
\text { Capriata }\end{array}$ & $\begin{array}{l}\text { Don Francisco Zapata, } \\
\text { XXIV }\end{array}$ & 4 \\
\hline 1602 & 160 & Milán & $\begin{array}{l}\text { Pablo Vicencio } \\
\text { Capriata }\end{array}$ & $\begin{array}{l}\text { Doctor Andrés Estacio, } \\
\text { médico }\end{array}$ & 5 \\
\hline
\end{tabular}

Estos ejemplos ilustran cómo las redes genovesas importaron espadas italianas para el mercado granadino. La documentación notarial, fragmentaria e incompleta para el caso granadino, nos ha dado una pista a seguir en los archivos italianos - Génova - para completar el tráfico armamentístico entre las dos penínsulas del Mediterráneo occidental.

¿Acaso fueron los genoveses los únicos que realizaron este comercio en Granada? Únicamente encontramos un caso donde el mercader de armas es castellano. Se trata del soriano Francisco de Juano, vecino de Yanguas, que llevó en 1584 a Granada "cuatro docenas de guarniciones de espadas de Durango del maestro que se dice Pedro de Ortuza ${ }^{78}$ " a cambio de 42 reales la docena ${ }^{79}$. Durango en esta época destacaba en la producción de estos elementos ${ }^{80}$. Nuevas investigaciones en los archivos podrán dibujar ésta y otras redes castellanas del comercio de espadas y otras armas.

\section{CONCLUSIONES}

Los envíos localizados en el Archivio di Stato di Firenze dejan clara la existencia de un comercio de espadas, desde los puertos del sur y levante con el puerto de Livorno que abasteció al mercado italiano. No obstante, necesitamos estudios en la documentación de dichos puertos para delimitar el origen de las mismas. Imaginamos que debían ser de muy buena calidad, pues todo apunta a que el mercado italiano estaba lleno de espadas de calidades medias y bajas. ¿Acaso las famosas espadas toledanas supusieron la mayoría de estos envíos? La preponderancia del puerto de Alicante como origen de estos fletes podría apuntar a este origen por la relación entre el puerto levantino con las ciudades castellanas del interior - Toledomientras que un origen en los puertos de Cádiz o Sevilla podría sugerir espadas del mundo atlántico (Bilbao, Alemania, Holanda o Francia).

Las redes mercantiles que intervienen en este comercio son variadas. La mayoría son mercaderes florentinos - si bien conocemos que algunos eran factores de los genoveses - y solo dos son castellanos, todos ellos con contactos internacionales.

La participación de los mercaderes genoveses en la importación de espadas italianas - Milán, Serravalle, Brescia, Génova - que queda puesta de manifiesto en las ciudades de Toledo, Valencia y Granada - Mayolo, Escalla, Capriata y Chavarino entre otros-, podrá se-

7 Seguramente su apellido sería “Ortuzar" bastante extendido en Durango.

AHPG, G-251: 238 (20-VIII-1584)

Dueñas Beraiz, 2004: 254. 
guramente extenderse a otras poblaciones del Sur y Levante peninsular con estudios similares en protocolos notariales de dichos lugares.

Tenemos abundantes noticias de los espaderos granadinos. Su número, actividad y sus enlaces con mercaderes de espadas genoveses, hace que nos planteemos hasta qué punto fueron importantes en el mercado de espadas castellano de la época. Nuevos estudios podrán situar a este colectivo en el mercado de espadas de la España de los Austrias.

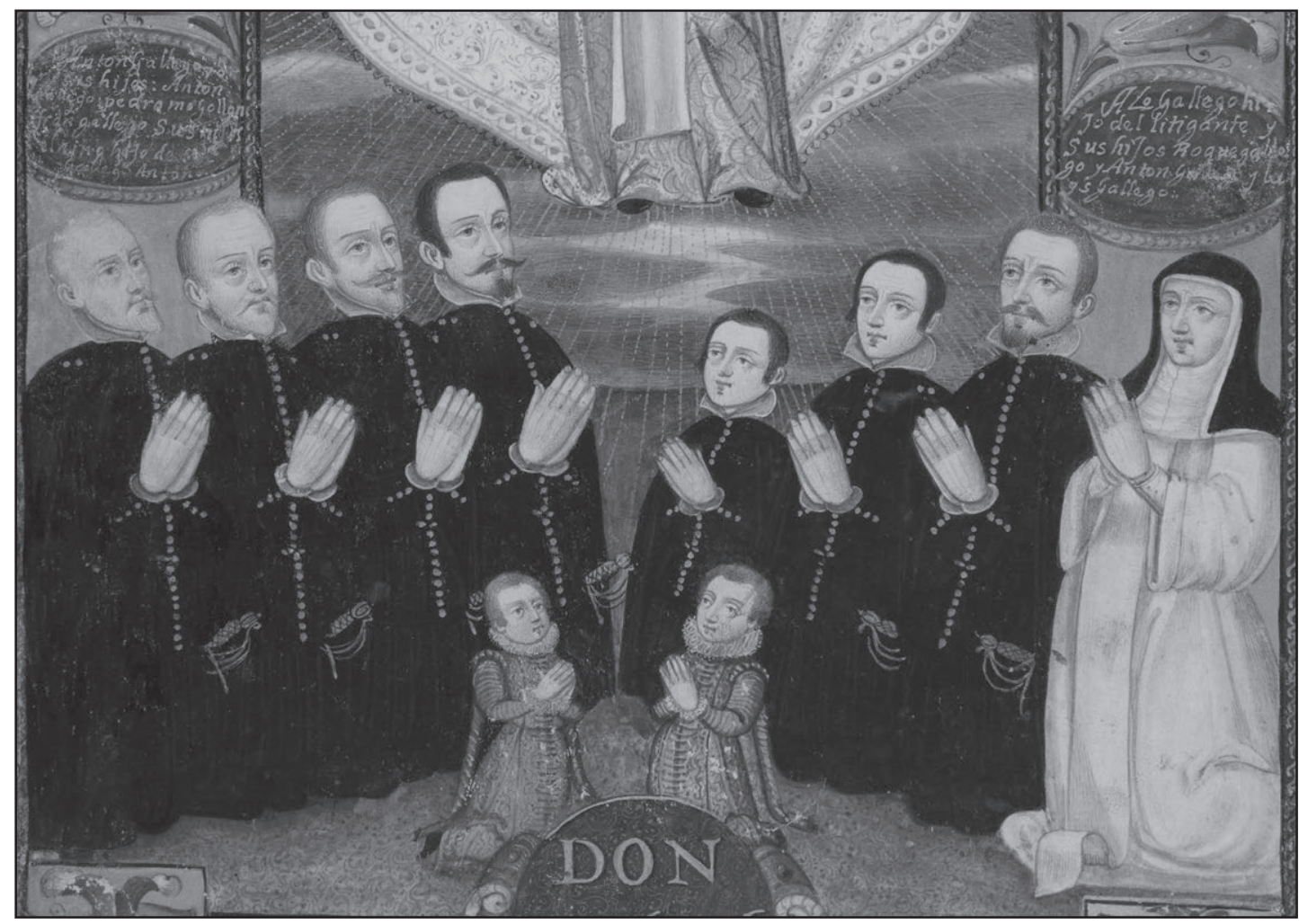

Figura 3. Detalle de una miniatura en la Carta Ejecutoria de Hidalguía a pedimento de Antón Gallego. Granada, 1626. Biblioteca Valenciana Digital. Todos los varones adultos portan espadas roperas.

\section{BIBLIOGRAFÍA}

Alloza Aparicio, A. (2003): «La Junta del Almirantazgo y la lucha contra el contrabando, 1625-1643». Espacio, Tiempo y Forma, Serie IV, Historia Moderna, 16: 217-254. http://dx.doi.org/10.5944/ etfiv.16.2003.3444

Alvar Ezquerra, A. (1996): «Los intercambios culturales entre los Países Bajos y Castilla en tiempos de Felipe II: un muestreo de las "cédulas de paso"». Indagación, 2: 91-109.

Azpiazu Elorza, J. A. (1994): «Fabricación y comercialización de armas en el valle de Deba (15501600)». Vasconia: Cuadernos de historia-geografía, 22: 9-72.

Barbiroli B. (2012): Repertorio storico degli archibugiari italiani dal XIV al XX secolo, Bologna.

Belfanti, C. M. (1993): «Rural Manufactures and Rural Proto-industries in the 'Italy of the Cities' from the Sixteenth through the Eighteenth Century». Continuity and Change, 2: 253-280.

Belfanti, C. M. (1998): «A chain of skills: the production cycle of firearms manufacture in the Brescia area from the sixteenth to the eighteenth centuries». en A. Guenzi, P. Massa and F. Piola Caselli (eds), Guilds, Markets and Work Regulations in Italy, 16th-19th Centuries, London: 266-283. 
Bernadet, J. (1891): Apuntes Arqueológicos. Armas y Armaduras. Las espadas de Toledo. Notas para la historia de la estatuaria movible en España, Toledo.

Blair, C. (1970): «A Royal Swordsmith and Damascener: Diego de Çaias». Metropolitan Museum Journal, 3: 149-198. http://dx.doi.org/10.2307/1512603

Braudel, F. y Romano, R. (1951): Navires et Marchandises à l'lentrée du port de Livourne (1547-1611), Paris.

Cano de Gardoqui, J. L. (2000): «Los inventarios de bienes de Don Juan de Austria. Mentalidad, gusto y vida material». M. J. Redondo y M. A. Zalama (coords.), Carlos Vy las Artes. Promoción artística y familia imperial. Valladolid: 323-351.

Carrión Arregui, I. M. (1998): «La crisis del siglo XVII y la producción de armamento en Gipuzkoa». Revista de Dirección y Administración de Empresas, 7: 21-31.

Casado Alonso, H. (2003): «El comercio del hierro vasco visto a través de los seguros marítimos burgaleses (1565-1596)». Itsas Memoria. Revista de Estudios Marítimos del País Vasco, 4: 165-192.

Chancillería de Granada, (1672): Ordenanzas de los muy ilustres, y muy magníficos señores que en Granada mandaron guardar, para la buena gobernación de su República, impresas en el año de 1552, que se han vuelto a imprimir por mandato de los señores Presidente y oidores de la Real Chancillería de esta ciudad de Granada, año de 1670, añadiendo otras que no estaban impresas, Granada.

Dueñas Beraiz, G. (2000): «Julián del Rey: Nuevos datos sobre su figura». Gladius, 20: 269-284. http:// dx.doi.org/10.3989/gladius.2000.74

Dueñas Beraiz, G. (2001): «La producción de armas blancas en Bilbao durante el siglo XVI». Gladius, 21: 269-290. http://dx.doi.org/10.3989/gladius.2001.90

Dueñas Beraiz, G. (2003): «Francisco de Santiago y Palomares y los espaderos toledanos». Gladius, 23: 269-306. http://dx.doi.org/10.3989/gladius.2003.52

Dueñas Beraiz, G. (2004): «Introducción al estudio tipológico de las espadas españolas: siglos XVIXVII». Gladius, 24: 209-260. http://dx.doi.org/10.3989/gladius.2004.42

Fernández González, F. (2000): Comerciantes vascos en Sevilla, 1650-1700, Sevilla.

Fernández Vega, P. (1935): «Dagas granadinas». Anuario del Cuerpo Facultativo de Archiveros Bibliotecarios y Arqueólogos, 3: 359-371.

Ferrandis Torres, J. (1943): «Espadas granadinas de la jineta». Archivo español de arte, 16, 57: 142-166.

Filippini, J. P. (1998), Il porto di Livorno e la Toscana: 1676-1814, Napoli.

Girón Pascual, R. M. (2013): Las Indias de Génova. Mercaderes genoveses en el reino de Granada durante la Edad Moderna. Tesis doctoral inédita. Granada.

Goldberg, E. L. (1996): «Relations between the Medici and the Spanish Courts, 1587-1621». The Burlington Magazine, 138, 1115: 105-114 y 1121: 529-540.

González Ramos, R. (2014): «La colección de armas de Íñigo López de Mendoza, V duque del Infantado». Gladius, 34: 153-198. http://dx.doi.org/10.3989/gladius.2014.0008

Ghezzi, R. (2007): Livorno e il mondo islamico nel XVII secolo: naviglio e comercio di importazione, Bari.

Lanero Fernández, J. (2008): «De cómo los ingleses deben comerciar en la península Ibérica: The Marchants Avizo (1589)». Pecvnia, 7: 149-205. http://dx.doi.org/10.18002/pec.v0i7.692

Larrañaga, R. (1981): Síntesis Histórica de la Armería Vasca, San Sebastián.

Larrañaga, R. (1984): «Espaderos vascos en Toledo». Vasconia: Cuadernos de historia-geografía, 4: 29-34.

Larrañaga, R. (1988): «El inventario de bienes de un armero del siglo XVI». Vasconia: Cuadernos de historia-geografía, 10: 91-100.

Leguina, E. (1885): La Espada. Apuntes para su historia en España. Sevilla.

Leguina, E. (1897): Los maestros espaderos, Sevilla.

Leguina, E. (1912): Glosario de voces de Armería, Madrid.

Lobo Cabrera, M. (2015): El comercio canario europeo bajo Felipe II, Alicante.

López de Coca, J. E. y López Beltrán, M. T. (1980): «Mercaderes genoveses en Málaga (1487-1516): los hermanos Centurión e Ytalián», Historia. Instituciones. Documentos, 7: 95-124.

López-Salazar Pérez, J. (2005): «El mundo rural en La Mancha cervantina: Labradores e hidalgos». Porfirio Sanz Camañes (ed.), La monarquía hispánica en tiempos del Quijote, Madrid: 15-62. 
Mark, P. y Silva Horta, J. (2011): The Forgotten Diaspora: Jewish Communities in West Africa and the Making of the Altlantic Word, Cambridge.

Martínez Enamorado, V. (2005): «La espada de protocolo del sultán Nazarí Muhammad V». Gladius, 25: 285-310. http://dx.doi.org/10.3989/gladius.2005.32

Mena García, M.C. (1998), Sevilla y las flotas de Indias: La Gran Armada de Castilla del Oro (15131514), Sevilla.

Montojo Montojo, V. (1993): El siglo de oro en Cartagena (1480-1640), Murcia.

Montojo Montojo, V. (2011): «El comercio de Alicante en el reinado de Carlos II». Saitabi, 60-61: 327 345.

Muñoz Navarro, D. (2010): «Relaciones comerciales entre el Reino de Valencia y el Norte de Italia en el tránsito del siglo XVI al XVII». RiMe. Rivista dell'Instituto di Storia dell 'Europa Mediterranea, 4: 319-334.

Norman, A. V. B. (1980): The rapier and Small-sword 1460-1820, Londres, 1980.

Otte, E. (1996): Sevilla y sus mercaderes a fines de la Edad Media, Sevilla.

Peláez Valle, J. M. (1983): «La espada ropera española en los siglos XVI y XVII». Gladius, 16: 147199.

Peláez Valle, J. M. (1986): «Comentarios metalúrgicos a la tecnología de procesos de elaboración del acero de las espadas de Toledo descritas en el documento de Palomares de 1772». Gladius, 17: 129-155.

Pérez Villalba, M. T. (2012): «Franceses en la Valencia del siglo XVI: Apuntes acerca del proceso de integración y trayectoria ejemplar del mercader Joan Augier». Estudis, 38: 323-345.

Rodríguez de Gracia, H. (2005a): «Las expectativas de los genoveses toledanos en tiempos del Quijote». Porfirio Sanz Camañes (ed.), La monarquía hispánica en tiempos del Quijote, Madrid: 165-187.

Rodríguez de Gracia, H. (2005b): «Mercaderes y financieros. Los genoveses de Toledo en 1561 y 1621». María Begoña Villar García y Pilar Pezzi Cristóbal, Los extranjeros en la España Moderna, Madrid: 597-610.

Ruiz Martín, F. (1965): Lettres marchandes échangées entre Florence et Medina del Campo, Paris.

Ruiz Martín, F. (1968): «Movimientos demográficos y económicos en el reino de Granada durante la segunda mitad del siglo XVI». Anuario de Historia Económica y Social, 1, 1: 127-183.

San Ruperto Albert, J. (2013): «Familia, redes mercantiles y poder en el siglo XVII: La llegada al reino de Valencia de los Cernesio». Tiempos Modernos, 27, 2.

Torrealba Domínguez, J. G. (1992): «El comercio no institucionalizado en Logroño, 1650-1750». Berсео, 123: 99-210.

Velasco Hernández, F. (2001): Auge y estancamiento de un enclave mercantil en la periferia. El nuevo resurgir de Cartagena entre 1540 y 1676, Murcia.

Viñas Mey, C. (1968): «Cuadro económico-social de la España de 1627-1628. Pragmáticas sobre tasas de las mercaderías y mantenimientos, jornales y salarios». Anuario de Historia Económica y Social, 1, 1: 715-772.

Wagner, E. (1969): Cut and Thrust Weapons.

Williams, A. (2012): The sword and the Crucible: A History of the Metallurgy of European Swords Up to the 16th Century, Boston.

Recibido: 22/06/2015

Aceptado: 11/04/2016 


\section{ANEXO I}

Tabla 7. Nómina de espaderos granadinos en el siglo XVI (1543-1604) ${ }^{81}$

\begin{tabular}{|l|l|c|}
\hline Espadero & Parroquia & Año \\
\hline Sebastián Hernández & Magdalena & 1543 \\
\hline Jaime Lafonte & Magdalena & $1543-1555$ \\
\hline Gregorio Rodríguez & Magdalena & 1547 \\
\hline Francisco Rodríguez & Magdalena & $1547-1550$ \\
\hline Francisco Gómez & Magdalena & 1550 \\
\hline Juan de Hita & Magdalena & 1550 \\
\hline Bartolomé Fernández & Magdalena & $1549-1550$ \\
\hline Diego Fernández & Magdalena & $1551-1553$ \\
\hline Diego Mexia & & 1569 \\
\hline Pedro de Torres & Iglesia Mayor & $1582-1604$ \\
\hline Juan García & Magdalena & $1550-1587$ \\
\hline Alonso Fernández & Magdalena & 1551 \\
\hline Cristóbal de Fustamante & & $1583-1600$ \\
\hline Luis de Aguilera & Magdalena & $1588-1604$ \\
\hline Cristóbal de Torres & Iglesia Mayor & 1588 \\
\hline Salvador Barrero & Magdalena & $1593-1604$ \\
\hline Justo de Flores & & 1604 \\
\hline Francisco Pérez & & 1604 \\
\hline Juan de Torres Colado & & 1604 \\
\hline Diego de Barrera & & 1604 \\
\hline Gaspar de los Reyes & & 1604 \\
\hline Luis García & & 1604 \\
\hline Nicolás de Fustamante & & 1604 \\
\hline Antonio Martínez & & 1604 \\
\hline Pedro de Ávila & & 1604 \\
\hline
\end{tabular}

\section{ANEXO II}

Tabla 8. Otros espaderos del reino de Granada (excepto la ciudad de Granada) ${ }^{82}$

\begin{tabular}{|l|l|l|}
\hline Espadero & Población & Años \\
\hline Miguel Hernández & Loja & 1583 \\
\hline Gaspar de Alarcón & Motril & 1587 \\
\hline Juan Navarro & Huéscar & 1598 \\
\hline Francisco García & Huéscar & 1605 \\
\hline Antonio de Tapia & Huéscar & 1611 \\
\hline
\end{tabular}

81 Fuente: BHR/Caja MS-2-047 y AHPG. Elaboración propia.

82 Fuente: AHPG, G-245: 102r; G-267: 501r; H-193: 24v; H-251: 956v; H-318: 100r. Elaboración propia. 